\title{
Polymeric mixed micelles loaded mitoxantrone for overcoming multidrug resistance in breast cancer via photodynamic therapy
}

This article was published in the following Dove Press journal:

International Journal of Nanomedicine

6 September 2017

Number of times this article has been viewed

\author{
Zeyong $\mathrm{Li}^{1, *}$ \\ Yuee $\mathrm{Cai}^{2}{ }^{2} *$ \\ Yiqiao Zhao' \\ Hua $\mathrm{Yu}^{2}$ \\ Haiyu Zhou ${ }^{3}$ \\ Meiwan Chen² \\ 'Department of Laboratory Medicine, \\ Guangdong No 2 Provincial People's \\ Hospital, Guangzhou, China; ${ }^{2}$ State \\ Key Laboratory of Quality Research \\ in Chinese Medicine, Institute of \\ Chinese Medical Sciences, University \\ of Macau, Macau, China; ${ }^{3}$ Department \\ of Thoracic Surgery, Guangdong \\ General Hospital, Guangdong \\ Academy of Medical Sciences, \\ Southern Medical University, South \\ China University of Technology, \\ Guangzhou, China \\ *These authors contributed equally \\ to this work
}

\begin{abstract}
Mitoxantrone (MIT) is an anticancer agent with photosensitive properties that is commonly used in various cancers. Multidrug resistance (MDR) effect has been an obstacle to using MIT for cancer therapy. Photochemical internalization, on account of photodynamic therapy, has been applied to improve the therapeutic effect of cancers with MDR effect. In this study, an MIT-poly( $\varepsilon$-caprolactone)-pluronic F68-poly( $\varepsilon$-caprolactone)/poly(D,L-lactideco-glycolide)-poly(ethylene glycol)-poly(D,L-lactide-co-glycolide) (MIT-PFP/PPP) mixed micelles system was applied to reverse the effect of MDR in MCF-7/ADR cells via photochemical reaction when exposed to near-infrared light. MIT-PFP/PPP mixed micelles showed effective interaction with near-infrared light at the wavelength of $660 \mathrm{~nm}$ and exerted great cytotoxicity in MCF-7/ADR cells with irradiation. Furthermore, MIT-PFP/PPP mixed micelles could improve reactive oxygen species (ROS) levels, decrease P-glycoprotein activity, and increase the cellular uptake of drugs with improved intracellular drug concentrations, which induced cell apoptosis in MCF-7/ADR cells under irradiation, despite MDR effect, as indicated by the increased level of cleaved poly ADP-ribose polymerase. These findings suggested that MIT-PFP/PPP mixed micelles may become a promising strategy to effectively reverse the MDR effect via photodynamic therapy in breast cancer.
\end{abstract}

Keywords: mitoxantrone, polymeric mixed micelles, multidrug resistance, photodynamic therapy, breast cancer

\section{Introduction}

Mitoxantrone (MIT, Figure 1A), a synthetic derivative of anthracenedione, is a topoisomerase II targeting drug with high efficacy in the treatment of various malignancies such as breast cancer and acute leukemia. ${ }^{1,2}$ Overexpression of adenosine triphosphate-binding cassette $(\mathrm{ABC})$ transporters is associated with resistance to different anticancer agents, including MIT, which hinders the clinical application of MIT for cancer treatment. ${ }^{2} \mathrm{P}$-glycoprotein (P-gp) is one of the ABC transporters and is overexpressed in $>40 \%$ of breast cancer cases. It participates in the active transport of anticancer drugs out of cancer cells, leading to decreased intracellular concentration of drugs and resulting in multidrug resistance (MDR) in clinical cases. ${ }^{3}$ Therefore, reversing the MDR effect on MIT in breast cancer has been a primary strategy to improve cancer therapy. Various methods have been applied to inhibit ABC transporters with the aim to improve the retention of anticancer drugs in cancer cells, including low-molecular weight P-gp inhibitors, polymer-drug conjugates, high-molecular weight polymer carriers, photodynamic therapy (PDT), and so on. ${ }^{4-6}$ 

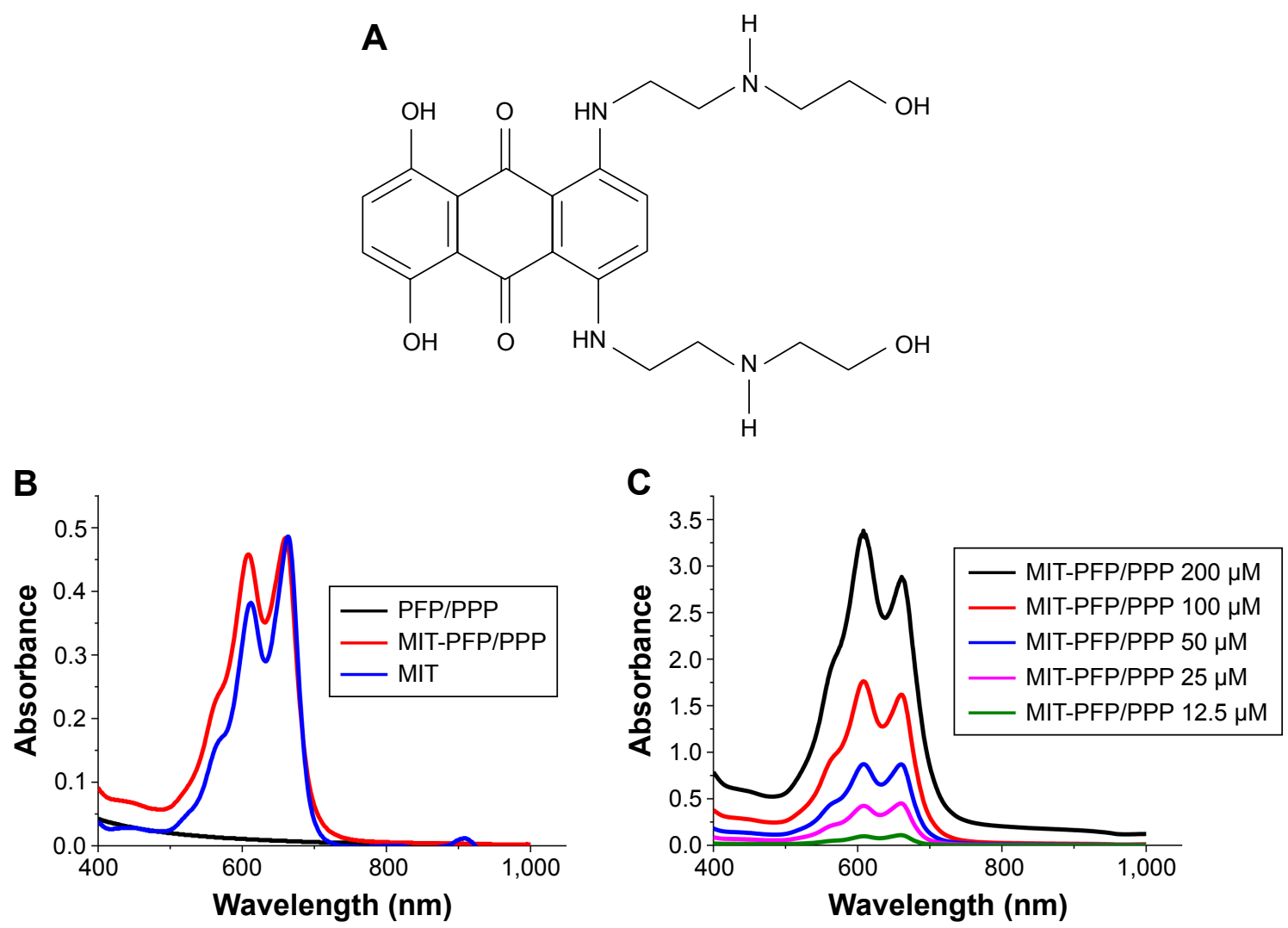

Figure I Chemical structure of MIT and photo absorption properties of MIT and MIT-PFP/PPP mixed micelles.

Notes: (A) The chemical structure of MIT; (B) photo absorption properties of MIT, MIT-PFP/PPP mixed micelles and blank PFP/PPP mixed micelles, measured by DR6000 UV-visible spectrophotometer at the wavelength range of $400-1,000 \mathrm{~nm}$; (C) photo absorption properties of MIT-PFP/PPP mixed micelles with different concentrations measured by DR6000 UV-visible spectrophotometer at the wavelength range of $400-1,000 \mathrm{~nm}$. These experiments were carried out in triplicate.

Abbreviations: MIT, mitoxantrone; PFP, poly(E-caprolactone)-pluronic F68-poly( $\varepsilon$-caprolactone); PPP, poly(D,L-lactide-co-glycolide)-poly(ethylene glycol)-poly(D,L-lactideco-glycolide).

PDT is a minimally invasive and clinically approved therapeutic modality with selective cytotoxicity against cancer. PDT requires three major components: photosensitizing agents, tissue oxygen, and laser irradiation at the wavelength of the photosensitizer. ${ }^{7}$ Photosensitizers kill cancer cells by generating reactive oxygen species (ROS) via transferring absorbed photon energy to oxygen molecules when the cells are exposed to irradiation with an appropriate wavelength. Photosensitizers are not toxic to cells without laser irradiation. ${ }^{8}$ PDT improves selectivity of anticancer agents and also decreases their side effects, compared to conventional radiotherapies and chemotherapies. ${ }^{9}$ Recently, photochemical internalization, an application of PDT, has been employed to improve the chemotherapeutic effect in cancers with MDR. Photochemical internalization destroys the cellular membrane with laser exposure by reactive oxygen-induced lipid perioxidation. ${ }^{6}$ MIT has two major absorption peaks at the wavelengths of 610 and $660 \mathrm{~nm}$, and it is an efficient photosensitizer to mediate cell death with exposure to light at the wavelength of $660 \mathrm{~nm} .{ }^{10}$ Studies of
PDT using MIT to treat breast cancer and melanoma cancer have been reported. ${ }^{11,12}$ To conquer the MDR effect of MIT in breast cancer cells, using the photosensitizing property of MIT may exert unexpected anticancer effect due to the generation of ROS in cancer cells.

Polymeric micelles formed by high-molecular weight polymers are capable of encapsulating hydrophobic drugs through their core-shell structure with favorable size and enhanced permeability and retention effect, of which some function as a biologic modifier against MDR. ${ }^{13,14}$ In our previous study, a mixed micelle system, formed by poly $(\varepsilon-$ caprolactone)-pluronic F68-poly(e-caprolactone) (PFP) and poly(D,L-lactide-co-glycolide)-poly(ethylene glycol)-poly (D,L-lactide-co-glycolide) (PPP), has been designed to efficiently deliver the anticancer agent MIT, while reversing MDR in breast cancer. ${ }^{15}$ As mentioned above, the application of PDT could reverse the MDR effect in cancer cells. ${ }^{6}$ It is meaningful to investigate the photosensitizing property of a clinically used anticancer agent (MIT) as PDT against the cancer cells with MDR. In this study, the previously designed 
PFP/PPP mixed micelles were applied to deliver MIT (MIT-PFP/PPP mixed micelles) to evaluate the anticancer effect of MIT with exposure to near-infrared light on the MDR breast cancer cells, the MCF-7/ADR cells.

\section{Materials and methods Materials}

MIT (98\% purity) was obtained from Meilun Biology Technology Company (Dalian, China). Phosphate buffer saline (PBS), penicillin-streptomycin, fetal bovine serum (FBS), $0.25 \% \operatorname{trypsin} / 1 \mathrm{mM}$ ethylenediaminetetraacetic acid $(\mathrm{w} / \mathrm{v})$ and propidium iodide (PI) were obtained from Thermo Fisher Scientific (Waltham, MA, USA). Hoechst 33342, wortmannin, genistein, methyl- $\beta$-cyclodextrin, 2-deoxyglucose and 3-(4,5dimethyl-2-thiazolyl)-2,5-diphenyl tetrazolium bromide (MTT) were obtained from Sigma Aldrich (St Louis, MO, USA). ROS Assay Kit was obtained from Beyotime (Shanghai, China). The primary antibodies against GAPDH and poly ADP-ribose polymerase (PARP) were obtained from Cell Signaling Technology (Boston, MA, USA). MultiDrug Resistance Assay Kit was purchased from Cayman Chemical (Ann Arbor, MI, USA). The chemicals were all of analytical grade, and ultra-filtered water was obtained with a Milli-Q apparatus (EMD Millipore, Billerica, MA, USA).

\section{Cell lines and cell culture}

Doxorubicin-resistant MCF-7 cells, named MCF-7/ADR, were obtained by stepwise exposure of MCF-7 cells to doxorubicin with increasing concentrations as previously reported. ${ }^{16} \mathrm{MCF}-7$ cells were obtained from American Tissue Culture Collection. MCF-7/ADR cells were incubated in Dulbecco's Modified Eagle's Medium (DMEM) with 10\% FBS (v/v), $100 \mathrm{U} / \mathrm{mL}$ streptomycin and $100 \mu \mathrm{g} / \mathrm{mL}$ penicillin with $5 \% \mathrm{CO}_{2}$ at $37^{\circ} \mathrm{C}$.

\section{Absorption properties of MIT}

To obtain the light absorbance band of MIT, the absorption spectra of blank PFP/PPP mixed micelles, MIT and MITPFP/PPP mixed micelles were measured by DR6000 UVVisible Spectrophotometer (HACH, Loveland, CO, USA). Various concentrations of MIT-PFP/PPP mixed micelles $(12.5,25,50,100,200 \mu \mathrm{M})$ were detected by UV-Visible Spectrophotometer to obtain the MIT light absorption spectra. ${ }^{12}$ The experiments were carried out in triplicate.

\section{Preparation and characterization of MIT-PFP/PPP mixed micelles}

MIT-PFP/PPP mixed micelles were prepared by solvent evaporation as reported previously. ${ }^{15}$ Fourteen milligrams copolymers of PFP and PPP with the ratio of 1:2 were mixed with $1 \mathrm{mg}$ MIT. The mixtures were then dissolved in water miscible organic solvent $(800 \mu \mathrm{L})$ of methanol and acetonitrile $(1: 1, \mathrm{v} / \mathrm{v})$ under ultrasonication. When the copolymers were completely dissolved, the mixed solution was added drop-wise into pure water and stirred for $5 \mathrm{~h}$ until the methanol and acetonitrile were evaporated. The final solution was filtered using a $0.45 \mu \mathrm{M}$ Millipore filter to remove any large aggregate. Filtration isolated MIT-PFP/PPP micelles with particle size $<450 \mathrm{~nm}$. The whole procedure was performed in a dark room. The experiments were carried out in triplicate. The particle size of MIT-PFP/PPP mixed micelles was detected by dynamic light scattering with a Zetasizer Nano ZSP system. The drug concentration of MIT-PFP/ PPP mixed micelles was detected by Waters e2695 HPLC with a $\mathrm{C} 18$ reverse-phase liquid chromatography column $(250 \times 4.6 \mathrm{~mm})$ at a flow rate of $1 \mathrm{~mL} / \mathrm{min}$ and maximum absorption wavelength of $609 \mathrm{~nm}$. The mobile phase was methanol $/ 0.25 \%$ acetic acid $(50 / 50, \mathrm{v} / \mathrm{v})$.

\section{In vitro cytotoxicity by photodynamic treatment}

MTT assay was used to determine the cytotoxicity of MITPFP/PPP mixed micelles with or without irradiation at $660 \mathrm{~nm}$ in MCF-7/ADR cells. ${ }^{17}$ MCF-7/ADR cells were seeded in 96-well plates with $100 \mu \mathrm{L}$ medium at a density of $5 \times 10^{3}$ cells/well and then incubated for $24 \mathrm{~h}$. Cells were treated with MIT and MIT-PFP/PPP mixed micelles at various concentrations ranging from 2.5 to $20 \mu \mathrm{M}$. After incubation for $4 \mathrm{~h}$, the original medium was discarded and fresh medium containing $0.5 \%$ FBS was added with laser irradiation at the power of 6,12 and $24 \mathrm{~mW}$ for $0.5 \mathrm{~h}$ with a $660 \mathrm{~nm}$ fiber-coupled laser system (LOSBLD-0660-2W; Hi-Tech Optoelectronics Co., Ltd., Beijing, China). After incubation for $24 \mathrm{~h}$ at $37^{\circ} \mathrm{C}, 20 \mu \mathrm{L}$ MTT dye at a concentration of $5 \mathrm{mg} / \mathrm{mL}$ was used to replace the medium and cells were incubated for another $4 \mathrm{~h}$ to form formazan crystals via mitochondrial dehydrogenases. Formazan crystals were dissolved with dimethyl sulfoxide. A microplate reader (SpectraMax M5; Molecular Devices, LLC, Sunnyvale, CA, USA) was used to record the spectrophotometric absorbance at $570 \mathrm{~nm}$, which was analyzed to demonstrate relative cell viability. The experiments were carried out in triplicate.

\section{Measurement of ROS}

An ROS assay kit was used to determine the intracellular ROS levels of cells treated with MIT or MIT-PFP/PPP mixed micelles. ${ }^{18}$ Briefly, MCF-7/ADR cells were seeded in 12-well plates at a density of $8 \times 10^{4}$ cells/well and incubated for $24 \mathrm{~h}$. 
MIT and MIT-PFP/PPP mixed micelles at a concentration of $20 \mu \mathrm{M}$ were added to MCF-7/ADR cells. After incubation for $4 \mathrm{~h}$, fresh medium with $0.5 \%$ FBS was used to replace the medium and cells were irradiated with $660 \mathrm{~nm}$ fiber-coupled laser at a power of $24 \mathrm{~mW}$ for $0.5 \mathrm{~h}$. The cells were then stained with $10 \mu \mathrm{M} 2$,7-dichlorodi-hydrofluorescein diacetate and incubated at $37^{\circ} \mathrm{C}$ for $30 \mathrm{~min}$. Cells were washed with PBS three times and collected. Stained cells were analyzed by flow cytometry (BD FACS Canto ${ }^{\mathrm{TM}}$ ). The experiments were carried out in triplicate.

\section{P-gp activity assay}

A multidrug resistance assay kit (Cayman Chemical) was utilized to determine the activity of P-gp. ${ }^{19} \mathrm{MCF}-7 / \mathrm{ADR}$ cells at a density of $5 \times 10^{3}$ cells/well were seeded in 96-well flat clear-bottom black-wall microplates and then incubated for $24 \mathrm{~h}$. Then, the cells were treated with MIT and MIT-PFP/ PPP mixed micelles and irradiated with laser mentioned above. One hundred microliters MDR dye-loading solution was added to each well followed by incubation at $37^{\circ} \mathrm{C}$ for another $1 \mathrm{~h}$ in the dark. Intracellular fluorescence was measured by a microplate reader with excitation wavelength of $490 \mathrm{~nm}$ and emission wavelength of $525 \mathrm{~nm}$. The experiments were carried out in triplicate.

\section{Cellular uptake of MIT and MIT-PFP/PPP and its mechanisms}

The cellular uptake of MIT and MIT-PFP/PPP mixed micelles was determined by flow cytometry (BD Accuri), which could measure the fluorescence of MIT associated with the cells using FL4 channel. ${ }^{17,20} \mathrm{MCF}-7 / \mathrm{ADR}$ cells at a density of $1 \times 10^{5}$ cells/wells were seeded in 12 -well plates and cultured for $24 \mathrm{~h}$. Cells were treated with MIT and MIT-PFP/PPP mixed micelles for $2 \mathrm{~h}$, followed by laser irradiation as mentioned above. After incubation for another $1.5 \mathrm{~h}$, the cells were collected by trypsinization and washed twice with PBS. Cells were suspended with $0.5 \mathrm{~mL}$ PBS and measured with flow cytometry. The experiments were carried out in triplicate.

The mechanisms of cellular uptake were determined using various endocytic inhibitors. ${ }^{21}$ Briefly, MCF-7/ADR cells were seeded at a density of $1 \times 10^{5}$ cells/well in 12 -well plates and cultured for $24 \mathrm{~h}$. Then, the cells were preincubated in the presence of different endocytic inhibitors for $1 \mathrm{~h}$, followed by treatment with MIT and MIT-PFP/PPP mixed micelles at a concentration of $20 \mu \mathrm{M}$ for $2 \mathrm{~h}$. The endocytic inhibitors were added at the following concentrations: $10 \mu \mathrm{M}$ of wortmannin, $50 \mu \mathrm{M}$ of genistein, $5 \mathrm{mM}$ of methyl- $\beta$-cyclodextrin and $20 \mathrm{mM}$ of 2-deoxyglucose. The cells were irradiated as mentioned above. After incubation for another $1.5 \mathrm{~h}$, the cells were trypsinized and washed twice with PBS. The cells were suspended in $0.5 \mathrm{~mL}$ PBS and analyzed by flow cytometry. The experiments were carried out in triplicate.

\section{Assessment of cell apoptosis}

Hoechst 33342 staining assay was used to observe nuclear morphologic changes and chromosome condensation in MCF-7/ADR cells. ${ }^{22} \mathrm{MCF}-7 / A D R$ cells were cultured in 96-well plates at a density of $5 \times 10^{3}$ cells/well for $24 \mathrm{~h}$. MIT and MIT-PFP/PPP mixed micelles were added to the cells and irradiated with laser as mentioned above. After incubation for $24 \mathrm{~h}$ at $37^{\circ} \mathrm{C}, 4 \%$ paraformaldehyde was utilized to fix the cells for $15 \mathrm{~min}$ and they were washed with PBS. The cells were then stained with $1 \mu \mathrm{g} / \mathrm{mL}$ of Hoechst 33342 at $25^{\circ} \mathrm{C}$ for $20 \mathrm{~min}$ in darkness. Lastly, the cells were observed and imaged by Incell Analyzer 2000 (GE Healthcare Bio-Sciences Corp., Piscataway, NJ, USA), which was equipped with a 4',6-diamidino-2-phenylindole filter (excitation of $350 \mathrm{~nm}$ and emission of $455 \mathrm{~nm}$ ). The experiments were carried out in triplicate.

Apoptosis was evaluated using the Annexin V-fluorescein isothiocyanate/PI detection kit (BD Pharmingen). ${ }^{23} \mathrm{MCF}-7 /$ ADR cells were cultured at a density of $5 \times 10^{3}$ cells/well in six-well plates for $24 \mathrm{~h}$. The cells were treated with MIT and MIT-PFP/PPP mixed micelles and irradiated with a laser as mentioned above. After incubation for $24 \mathrm{~h}$ at $37^{\circ} \mathrm{C}$, the cells were collected by trypsinization, washed twice with ice-cold PBS and gently suspended in $100 \mu \mathrm{L}$ binding buffer containing $20 \mu \mathrm{g} / \mathrm{mL}$ Annexin V-fluorescein isothiocyanate stain for $30 \mathrm{~min}$, followed by staining with PI $(10 \mu \mathrm{L})$ for $5 \mathrm{~min}$. Cell apoptosis was measured by flow cytometry. The experiments were carried out in triplicate.

\section{Western blot analysis}

Western blot analysis was used to measure apoptosis of cells treated with MIT and MIT-PFP/PPP mixed micelles. ${ }^{24}$ Briefly, MIT and MIT-PFP/PPP mixed micelles were added to $\mathrm{MCF}-7 / \mathrm{ADR}$ cells and irradiated with laser as mentioned above. After the cells were incubated for $24 \mathrm{~h}$, total cellular proteins were obtained using radioimmunoprecipitation assay lysis buffer with 1\% phenylmethanesulfonylfluoride and $1 \%$ protease inhibitor cocktail (Thermo Fischer Scientific). The concentrations of total protein were determined by a bicinchoninic acid protein assay kit (Thermo Fischer Scientific). Then, 15\% sodium dodecylsulfate polyacrylamide gel electrophoresis was used to separate proteins and the corresponding proteins were transferred to polyvinylidene fluoride membranes $(0.22 \mu \mathrm{m})$. After being 
blocked for $1 \mathrm{~h}$ by $5 \%$ non-fat dried milk, the membranes were incubated with specific primary antibodies $(1: 1,000$; Cell Signaling Technology) against PARP and GAPDH, followed by incubation with secondary rabbit antibodies (1:1,000; Cell Signaling Technology). An electrochemiluminescence Advanced Western Blotting Detection Kit from GE Healthcare Bio-Sciences Corp. was used to visualize the protein bands. The experiments were carried out in triplicate.

\section{Statistical analysis}

The data were analyzed by one-way analysis of variance followed by Tukey's Multiple Comparison Test using GraphPad Prism 5 software (GraphPad Software, Inc., San Diego, CA, USA). The results were expressed as mean $\pm \mathrm{SD}$. $P$-value lower than 0.05 was considered statistically significant.

\section{Results and discussion}

\section{Absorption properties of MIT}

It was reported that MIT has two photo absorption peaks, one at $600 \mathrm{~nm}$ and the other at $660 \mathrm{~nm}$. MIT acts as a photosensitizer with laser irradiation at the wavelength of $660 \mathrm{~nm}$ to induce cell death. ${ }^{12}$ As shown in Figure 1B, both MIT and MIT-PFP/PPP mixed micelles have two major absorption peaks at 600 and $660 \mathrm{~nm}$, while PFP/PPP micelles have no specific absorption peak at the wavelength range of 400-1,000 $\mathrm{nm}$. This result indicated that the encapsulation of MIT by PFP/PPP mixed micelles had no influence in the absorption properties of MIT, enabling its effective interaction with near-infrared light at the wavelength of $660 \mathrm{~nm}$. Additionally, the photo absorption of MIT-PFP/PPP mixed micelles increases in a dose-dependent manner (Figure 1C).

\section{In vitro cytotoxicity by photodynamic treatment}

MTT assay was used to determine the cytotoxicity of MITPFP/PPP mixed micelles via PDT in MCF-7/ADR cells. As shown in Figure 2, cells treated with or without free MIT showed negligible cytotoxicity in MCF-7/ADR cells with or without irradiation ( 6 and $12 \mathrm{~mW}$ ) for $24 \mathrm{~h}$, indicating that free MIT has no obvious anticancer effect in MCF-7/ADR
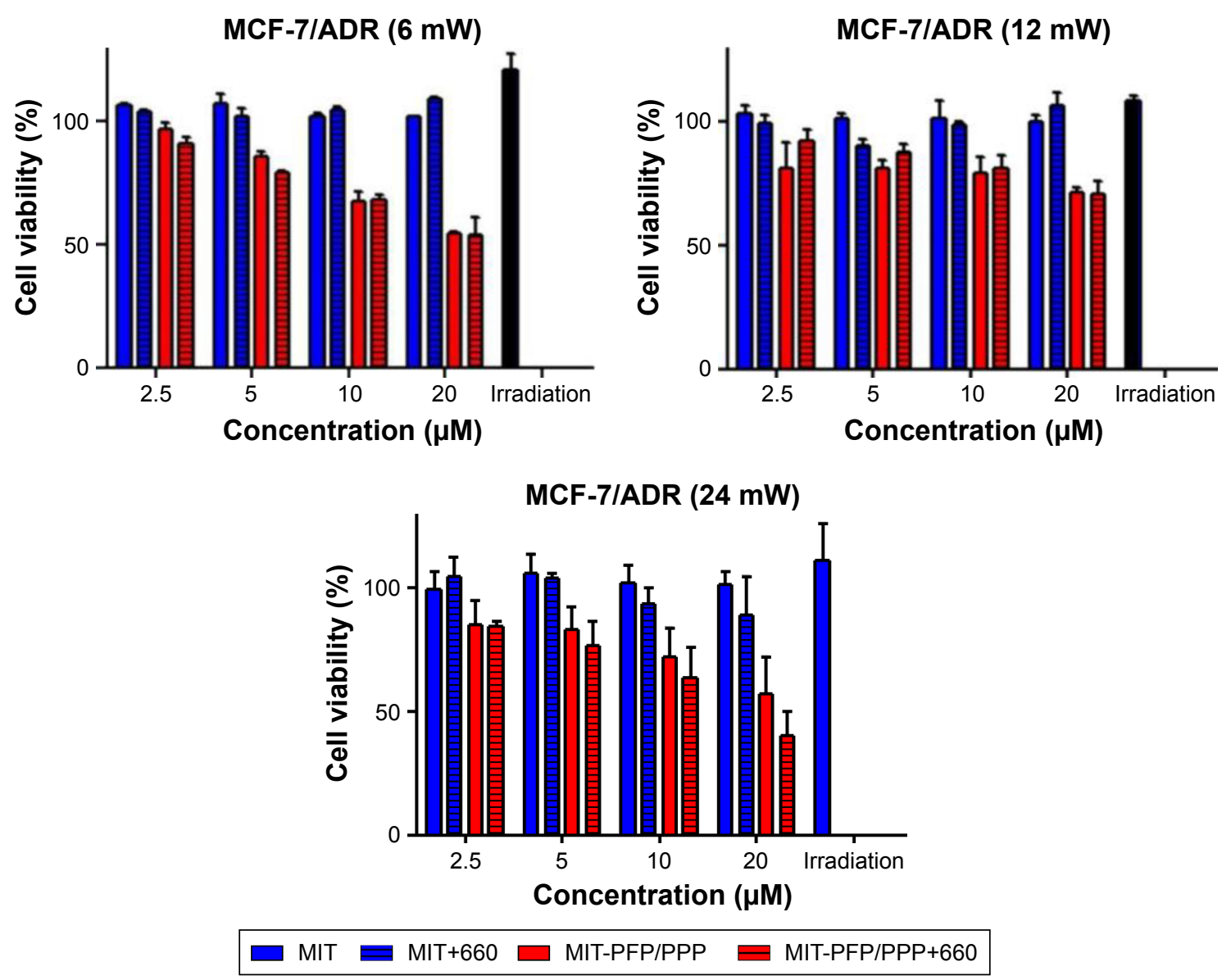

Figure 2 Cell viability of free MIT and MIT-PFP/PPP mixed micelles in MCF-7/ADR cells with or without laser irradiation at the power of 6,12 and 24 mW for $0.5 \mathrm{~h}$ with a $660 \mathrm{~nm}$ fiber-coupled laser system (mean $\pm \mathrm{SD}, \mathrm{n}=3$ ).

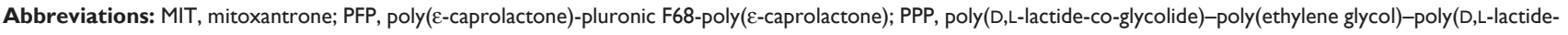
co-glycolide). 
cells. Meanwhile, MCF-7/ADR cells irradiated with different laser intensities $(6,12$ and $24 \mathrm{~mW}$ ) had no cytotoxicity. Cell viability reduced to around $60 \%$ in the cells treated with MIT-PFP/PPP mixed micelles $(20 \mu \mathrm{M})$ and irradiation at $24 \mathrm{~mW}$, while the reduction rates of cell viability at 6 and $12 \mathrm{~mW}$ were about $46 \%$ and $28.61 \%$, respectively. With irradiation, MCF-7/ADR cells treated with MIT (20 $\mu \mathrm{M})$ exhibited $11 \%$ cytotoxicity in MCF-7/ADR cells, which was much lower than that of MIT-PFP/PPP mixed micelles $(20 \mu \mathrm{M})$ with irradiation (60\%). Without irradiation, MCF-7/ADR cells treated with MIT-PFP/PPP mixed micelles $(20 \mu \mathrm{M})$ exerted $43 \%$ cytotoxicity, which was also lower than that of MIT-PFP/ PPP mixed micelles $(20 \mu \mathrm{M})$ with irradiation $(60 \%)$. These results indicate that PDT using MIT as a photosensitizer exerted strong anticancer effects under irradiation and MITPFP/PPP mixed micelles showed the highest cytotoxicity in MCF-7/ADR cells with irradiation among these treatments.

\section{Measurement of ROS}

PDT inhibited cell growth in cancer cells upon exposure to near-infrared irradiation through the generation of ROS. ${ }^{25}$ Whether the MIT-PFP/PPP mixed micelles generated ROS was measured in MCF-7/ADR cells under irradiation (24 mW). As shown in Figure 3A, MCF-7/ADR cells treated with MIT and MIT-PFP/PPP mixed micelles without irradiation exhibited negligible changes in ROS levels, while increased ROS levels were observed in the cells treated with both MIT and MIT-PFP/PPP mixed micelles after irradiation. These results
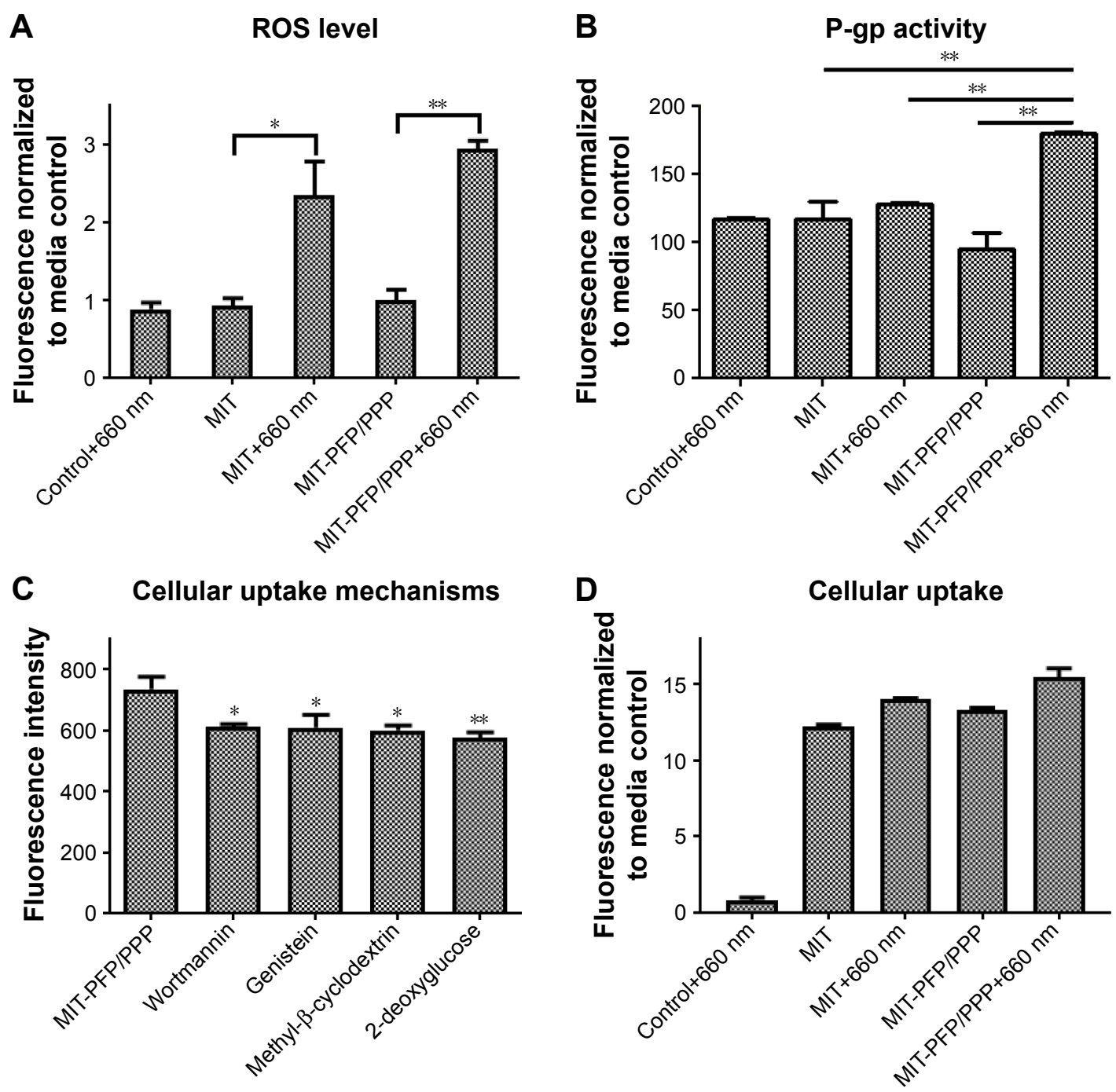

Figure 3 ROS levels, P-gP activity, cellular uptake mechanisms and cellular uptake of free MIT and MIT-PFP/PPP mixed micelles with or without irradiation at the power of $24 \mathrm{~mW}$ for $0.5 \mathrm{~h}$ with a $660 \mathrm{~nm}$ fiber-coupled laser system in MCF-7/ADR cells (mean \pm SD, $n=3$ ).

Notes: (A) ROS levels were determined by a ROS assay kit with fluorescent analysis by flow cytometry. (B) P-gP activity was detected by a multidrug resistance assay kit. (C) Cellular uptake mechanisms were determined by preincubation with different endocytic inhibitors and (D) intracellular uptake was detected by measuring the fluorescence of mitoxantrone associated with the cells by flow cytometry. $* P<0.05$ and $* * p<0.01$.

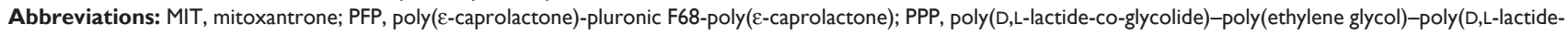
co-glycolide); P-gP, P-glycoprotein; ROS, reactive oxygen species. 
suggest that both free MIT and MIT-PFP/PPP mixed micelles may induce cytotoxicity in MCF-7/ADR cells via the production of ROS by photochemical reaction under irradiation.

\section{P-gp activity}

Cayman's MDR assay kit was used to determine P-gp activity. ${ }^{19}$ The kit used a cell-permeable nonfluorescent dye (calcein AM), which is cleaved intracellularly into a fluorescent molecule (calcein) for the detection of anticancer agents inhibiting MDR proteins. As shown in Figure 3B, P-gp activity was suppressed in MCF-7/ADR cells treated with MIT-PFP/PPP mixed micelles with irradiation, while there were no obvious changes on treating with MIT and MIT-PFP/PPP mixed micelles without irradiation. Meanwhile, there was also no obvious change in the P-gp activity of cells treated with MIT and irradiation, compared to that of cells treated with MIT and MIT-PFP/PPP mixed micelles without irradiation. This result demonstrated that MIT-PFP/ PPP mixed micelles with irradiation were able to suppress $\mathrm{P}$-gp activity in MCF-7/ADR cells.

\section{Cellular uptake of MIT-PFP/PPP mixed micelles and its mechanisms}

There might be different ways that cells can uptake MITPFP/PPP mixed micelles. We analyzed the internalization of
MIT-PFP/PPP mixed micelles by using different endocytic inhibitors. ${ }^{21}$ As shown in Figure 3C, there was a significantly lower cellular uptake with 2-deoxyglucose exposure, indicating that cellular uptake with MIT-PFP/PPP mixed micelles may be associated with an energy-dependent endocytosis. Methyl$\beta$-cyclodextrin and wortmannin exhibited a significant inhibitory effect, indicating that the internalization pathways were caveolae- and macropinocytosis-mediated pathways. Therefore, MIT-PFP/PPP mixed micelles could enter and stay in cancer cells while maintaining favorable drug concentration.

To investigate the ability of MIT-PFP/PPP mixed micelles to efficiently deliver anticancer drug to the cytosol in MCF-7/ ADR cells under laser irradiation, the cellular internalization of MIT-PFP/PPP mixed micelles was examined by flow cytometry. Figure 3D shows an increasing trend in the cellular uptake of MIT from groups (MIT, MIT+660 nm, MITPFP/PPP and MIT-PFP/PPP+660 nm) after incubation for $2 \mathrm{~h}$. This result suggested that MIT-PFP/PPP mixed micelles may improve intracellular concentration of anticancer drug under irradiation by photochemical reaction.

\section{Assessment of cell apoptosis}

Nuclear staining and Annexin V/PI staining were used to measure the apoptosis of MCF-7/ADR cells treated with MIT-PFP/PPP mixed micelles. As shown in Figure 4,
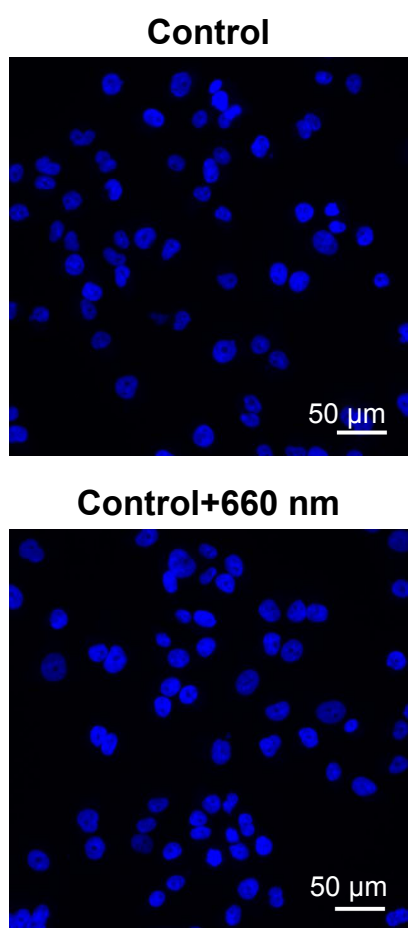

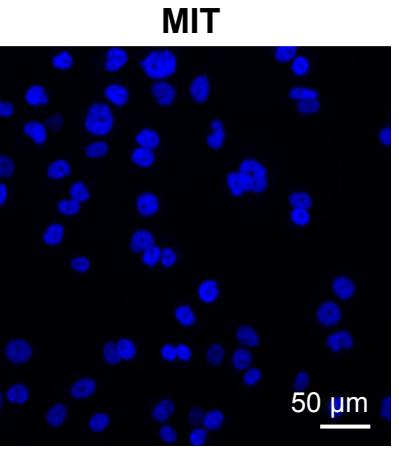

MIT+660 nm

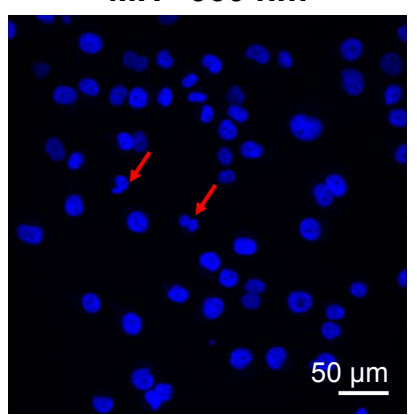

MIT-PFP/PPP

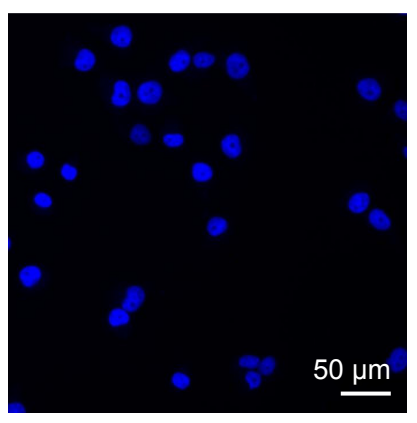

MIT-PFP/PPP+660 nm

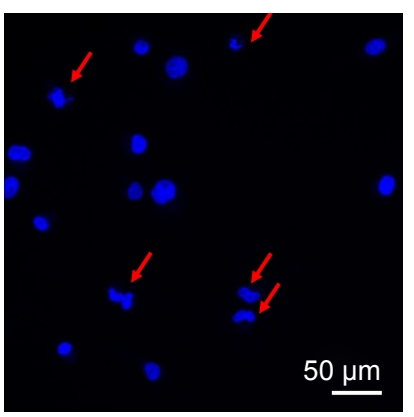

Figure 4 Nuclear staining by Hoechst 33342 in MCF-7/ADR cells treated with MIT and MIT-PFP/PPP mixed micelles with or without laser irradiation at the power of 24 mW for $0.5 \mathrm{~h}$ with a $660 \mathrm{~nm}$ fiber-coupled laser system.

Notes: The experiments were carried out in triplicate. The arrows indicate nuclear morphologic changes and chromosome condensation.

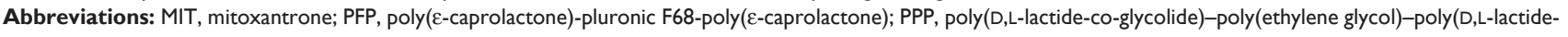
co-glycolide). 
MCF-7/ADR cells treated with MIT and MIT-PFP/PPP mixed micelles without irradiation were rounded and showed no nuclear condensation, indicating that there were no apoptotic cells. Interestingly, the number of MIT-PFP/PPP mixed micelles-treated MCF-7/ADR cells was less than the cells treated with free MIT, indicating the antiproliferative ability of MIT-PFP/PPP mixed micelles. After irradiation with laser, MCF-7/ADR cells treated with free MIT showed less cell apoptosis than the cells treated with MIT-PFP/PPP mixed micelles. In addition, MCF-7/ADR cells treated with
MIT-PFP/PPP mixed micelles had less proliferating cells. These results indicated that MIT-PFP/PPP mixed micelles exerted antiproliferative effect and induced cell apoptosis in MCF-7/ADR cells under irradiation.

To confirm the cell apoptosis of MIT-PFP/PPP mixed micelles under irradiation, Annexin V/PI staining was used to analyze apoptotic cells. As shown in Figure 5, about 7\% and $9 \%$ apoptotic cells were observed in the cells treated with MIT and MIT-PFP/PPP mixed micelles, respectively, while the cells treated with MIT and MIT-PFP/PPP mixed
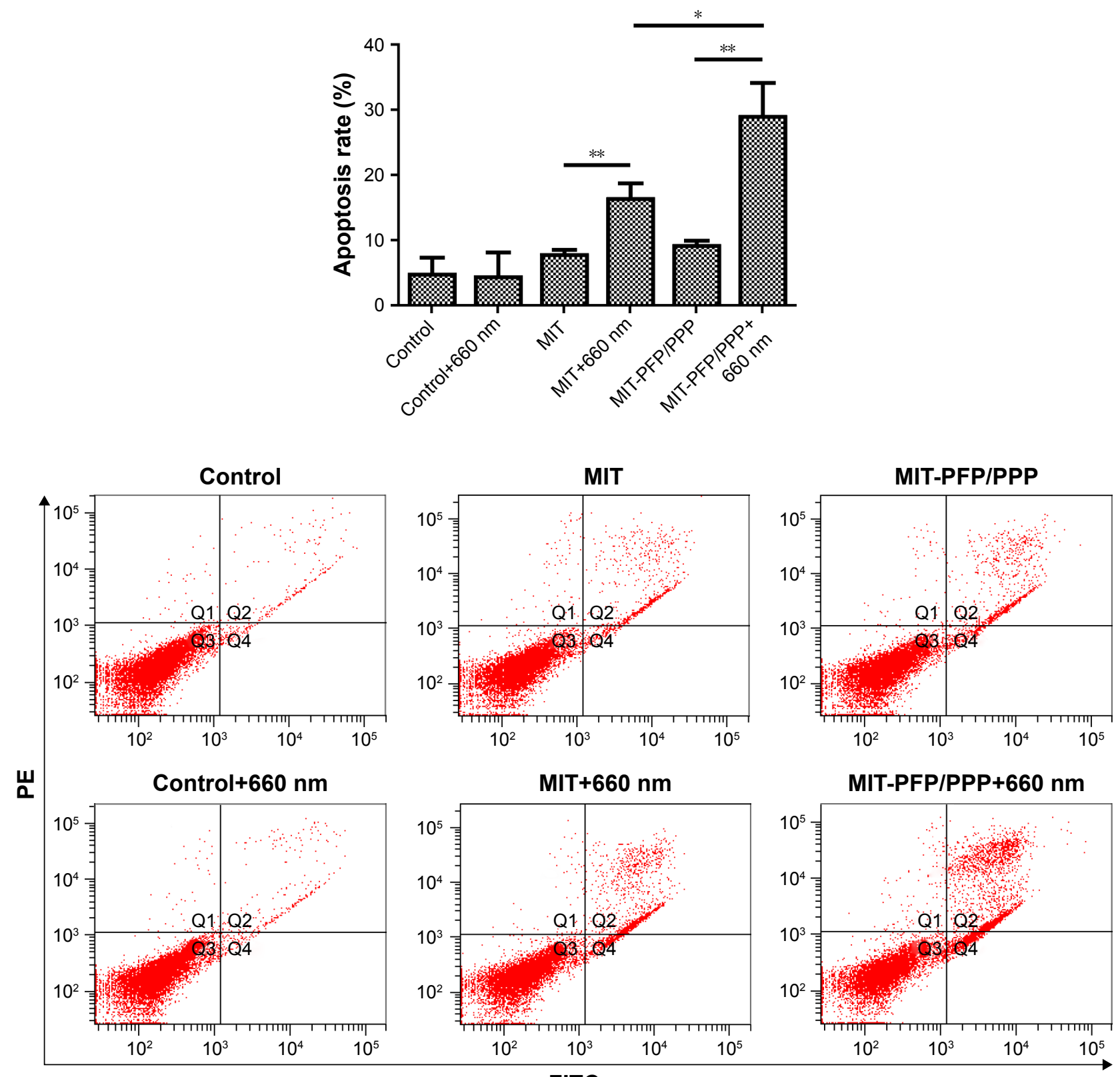

FITC

Figure 5 Apoptotic analysis by Annexin V-FITC/PI detection kit in MCF-7/ADR cells treated with MIT-PFP/PPP mixed micelles with or without laser irradiation at the power of $24 \mathrm{~mW}$ for $0.5 \mathrm{~h}$ with a $660 \mathrm{~nm}$ fiber-coupled laser system (mean $\pm S D, \mathrm{n}=3$ ).

Note: $* P<0.05$ and $* * P<0.01$.

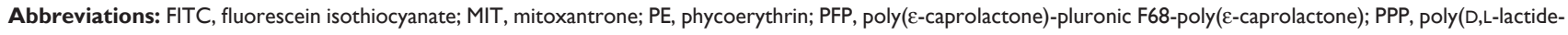
co-glycolide)-poly(ethylene glycol)-poly(D,L-lactide-co-glycolide). 


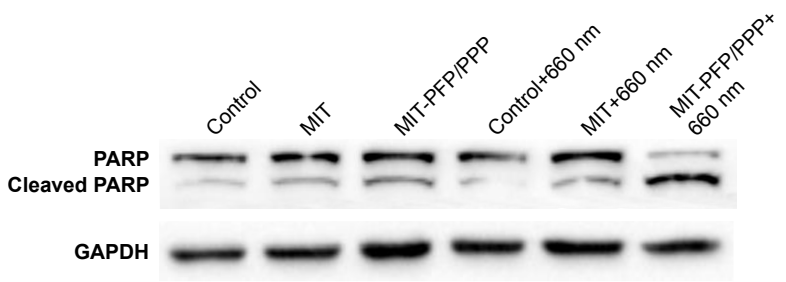

Figure 6 Western blot analysis of PARP expression in MCF-7/ADR cells treated with MIT and MIT-PFP/PPP mixed micelles with or without laser irradiation at the power of $24 \mathrm{~mW}$ for $0.5 \mathrm{~h}$ with a $660 \mathrm{~nm}$ fiber-coupled laser system.

Note: The experiments were carried out in triplicate.

Abbreviations: MIT, mitoxantrone; PARP, poly ADP-ribose polymerase; PFP,

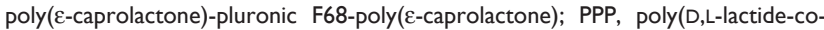
glycolide)-poly(ethylene glycol)-poly(D,L-lactide-co-glycolide).

micelles with irradiation showed $16 \%$ and $29 \%$ apoptosis, respectively. MIT-PFP/PPP mixed micelles with irradiation exhibited the highest cell apoptotic rate $(29 \%)$. These results demonstrated that MIT-PFP/PPP mixed micelles could induce cell apoptosis in MCF-7/ADR cells upon irradiation.

\section{Analysis of apoptosis-related biomarker by Western blot}

Western blot analysis was used to examine the expression of biomarker related to cell apoptosis in MIT-PFP/PPP mixed micelles-treated MCF-7/ADR cells. PARP was chosen because it is related to cell death and is specifically cleaved in apoptotic cells. As shown in Figure 6, the level of cleaved PARP in cells treated with MIT-PFP/PPP mixed micelles was increased under irradiation, indicating that MIT-PFP/ PPP mixed micelles with irradiation induced apoptosis in MCF-7/ADR cells by increasing cleaved PARP.

\section{Conclusion}

In this study, MIT-PFP/PPP mixed micelles system was used to reverse MDR in MCF-7/ADR breast cancer cells by PDT. After irradiation, MIT-PFP/PPP micelles were able to increase ROS levels, decrease P-gp activity and increase cellular uptake of MIT-PFP/PPP mixed micelles, which induced cell apoptosis and reversed the effect of MDR. Meanwhile, the level of cleaved PARP was increased by treatment with MIT-PFP/PPP mixed micelles, indicating cell apoptosis under irradiation by PDT in MCF-7/ADR cells. Furthermore, it is necessary to perform further study in vivo. Collectively, MIT-PFP/PPP mixed micelles may provide a promising strategy to efficiently reverse MDR by PDT in breast cancer.

\section{Acknowledgments}

This study was supported by the National Natural Science Foundation of China (81403120), the Guangdong Province
Medical Scientific Research Foundation (A2015333 and B2010004), and the Guangdong Province Science and Technology Planning Project (2014A020212406 and 2016ZC0158).

\section{Disclosure}

The authors report no conflicts of interest in this work.

\section{References}

1. Komeili-Movahhed T, Fouladdel S, Barzegar E, et al. PI3K/Akt inhibition and down-regulation of BCRP re-sensitize MCF7 breast cancer cell line to mitoxantrone chemotherapy. Iran J Basic Med Sci. 2015;18(5):472-477.

2. Hou L, Feng Q, Wang Y, et al. Multifunctional hyaluronic acid modified graphene oxide loaded with mitoxantrone for overcoming drug resistance in cancer. Nanotechnology. 2016;27(1):015701.

3. Yan CS, Wong IL, Chan KF, et al. A new class of safe, potent, and specific P-gp modulator: flavonoid dimer FD18 reverses P-gp-mediated multidrug resistance in human breast xenograft in vivo. Mol Pharm. 2015; 12(10):3507-3517.

4. Braunova A, Kostka L, Sivak L, et al. Tumor-targeted micelle-forming block copolymers for overcoming of multidrug resistance. J Control Release. 2017;245:41-51.

5. Zhang J, Zhao X, Chen Q, et al. Systematic evaluation of multifunctional paclitaxel-loaded polymeric mixed micelles as a potential anticancer remedy to overcome multidrug resistance. Acta Biomater. 2017;50: 381-395.

6. Park H, Park W, Na K. Doxorubicin loaded singlet-oxygen producible polymeric micelle based on chlorine e6 conjugated pluronic F127 for overcoming drug resistance in cancer. Biomaterials. 2014;35(27) 7963-7969.

7. Agostinis P, Berg K, Cengel KA, et al. Photodynamic therapy of cancer: an update. CA Cancer J Clin. 2011;61(4):250-281.

8. Wang S, Huang P, Nie L, et al. Single continuous wave laser induced photodynamic/plasmonic photothermal therapy using photosensitizerfunctionalized gold nanostars. Adv Mater. 2013;25(22):3055-3061.

9. Huang $\mathrm{P}, \mathrm{Xu} \mathrm{C}$, Lin J, et al. Folic acid-conjugated graphene oxide loaded with photosensitizers for targeting photodynamic therapy. Theranostics. 2011;1:240-250.

10. Sazgarnia A, Montazerabadi AR, Bahreyni-Toosi MH, Ahmadi A, Aledavood A. In vitro survival of MCF-7 breast cancer cells following combined treatment with ionizing radiation and mitoxantronemediated photodynamic therapy. Photodiagnosis Photodyn Ther. 2013; 10(1):72-78.

11. Sazgarnia A, Montazerabadi AR, Bahreyni-Toosi MH, Ahmadi A. Photosensitizing and radiosensitizing effects of mitoxantrone: combined chemo-, photo-, and radiotherapy of DFW human melanoma cells. Lasers Med Sci. 2013;28(6):1533-1539.

12. Montazerabadi AR, Sazgarnia A, Bahreyni-Toosi MH, Ahmadi A, Shakeri-Zadeh A, Aledavood A. Mitoxantrone as a prospective photosensitizer for photodynamic therapy of breast cancer. Photodiagnosis Photodyn Ther. 2012;9(1):46-51.

13. Alvarez-Lorenzo C, Sosnik A, Concheiro A. PEO-PPO block copolymers for passive micellar targeting and overcoming multidrug resistance in cancer therapy. Curr Drug Targets. 2011;12(8):1112-1130.

14. Kabanov AV, Batrakova EV, Miller DW. Pluronic block copolymers as modulators of drug efflux transporter activity in the blood-brain barrier. Adv Drug Deliv Rev. 2003;55(1):151-164.

15. Cai Y, Wang S, Wu M, et al. PCL-F68-PCL/PLGA-PEG-PLGA mixed micelles mediated delivery of mitoxantrone for reversing multidrug resistant in breast cancer. RSC Adances. 2016;6:35318-35327.

16. Wang S, Wang L, Shi Z, Zhong Z, Chen M, Wang Y. Evodiamine synergizes with doxorubicin in the treatment of chemoresistant human breast cancer without inhibiting P-glycoprotein. PLoS One. 2014; 9(5):e97512. 
17. Ding F, Li HJ, Wang JX, et al. Chlorin e6-encapsulated polyphosphoester based nanocarriers with viscous flow core for effective treatment of pancreatic cancer. ACS Appl Mater Interfaces. 2015;7(33): 18856-18865.

18. Hao Y, Zhang B, Zheng C, et al. Multifunctional nanoplatform for enhanced photodynamic cancer therapy and magnetic resonance imaging. Colloids Surf B Biointerfaces. 2017;151:384-393.

19. Wang S, Wang L, Chen M, Wang Y. Gambogic acid sensitizes resistant breast cancer cells to doxorubicin through inhibiting P-glycoprotein and suppressing survivin expression. Chem Biol Interact. 2015;235: 76-84.

20. Perillo E, Allard-Vannier E, Falanga A, et al. Quantitative and qualitative effect of gH625 on the nanoliposome-mediated delivery of mitoxantrone anticancer drug to HeLa cells. Int J Pharm. 2015;488(1-2):59-66.

21. Chen F, Zhang J, He Y, Fang X, Wang Y, Chen M. Glycyrrhetinic aciddecorated and reduction-sensitive micelles to enhance the bioavailability and anti-hepatocellular carcinoma efficacy of tanshinone IIA. Biomater Sci. 2016;4(1):167-182.
22. Wang S, Chen R, Morott J, Repka MA, Wang Y, Chen M. mPEG-b-PCL/ TPGS mixed micelles for delivery of resveratrol in overcoming resistant breast cancer. Expert Opin Drug Deliv. 2015;12(3):361-373.

23. Zhang J, Zhang M, Ji J, et al. Glycyrrhetinic acid-mediated polymeric drug delivery targeting the acidic microenvironment of hepatocellular carcinoma. Pharm Res. 2015;32(10):3376-3390.

24. Wang S, Yang Y, Wang Y, Chen M. Gambogic acid-loaded pH-sensitive mixed micelles for overcoming breast cancer resistance. Int J Pharm. 2015;495(2):840-848.

25. Pais-Silva C, de Melo-Diogo D, Correia IJ. IR780-loaded TPGSTOS micelles for breast cancer photodynamic therapy. Eur J Pharm Biopharm. 2017;113:108-117.
International Journal of Nanomedicine

\section{Publish your work in this journal}

The International Journal of Nanomedicine is an international, peerreviewed journal focusing on the application of nanotechnology in diagnostics, therapeutics, and drug delivery systems throughout the biomedical field. This journal is indexed on PubMed Central, MedLine, CAS, SciSearch ${ }^{\circledR}$, Current Contents ${ }^{\circledR} /$ Clinical Medicine,

\section{Dovepress}

Journal Citation Reports/Science Edition, EMBase, Scopus and the Elsevier Bibliographic databases. The manuscript management system is completely online and includes a very quick and fair peer-review system, which is all easy to use. Visit http://www.dovepress.com/ testimonials.php to read real quotes from published authors. 